\title{
Pre-attentive processing in children with early and continuously-treated PKU. Effects of concurrent Phe level and lifetime dietary control
}

\author{
Leo M. J. de Sonneville • Stephan C. J. Huijbregts • \\ Robert Licht • Joseph A. Sergeant • \\ Francjan J. van Spronsen
}

Received: 9 November 2010 /Revised: 8 March 2011 / Accepted: 21 March 2011 /Published online: 4 May 2011

(C) The Author(s) 2011. This article is published with open access at Springerlink.com

\begin{abstract}
Sixty-four children, aged 7 to 14 years, with early-treated PKU, were compared with control children on visual evoked potential (VEP) amplitudes and latencies and auditory mismatch negativity (MMN) amplitudes. It was further investigated whether indices of dietary control would be associated with these evoked potentials parameters. There were no significant differences between controls and children with PKU in VEP- and MMN-indices. However, higher lifetime Phe levels were, in varying degree and stronger than concurrent Phe level, related to increased N75 amplitudes, suggesting abnormalities in attention, and longer P110 latencies, indicating a reduction in speed of neural processing, possibly due to deficits in myelination or reduced dopamine levels in brain and retina. Similarly, higher lifetime Phe levels and Index of Dietary
\end{abstract}

Communicated by: Anita MacDonald

References to electronic databases: Phenylketonuria, OMIM 261600 and 261630 .

Competing interest: None declared.

L. M. J. de Sonneville $(\bowtie) \cdot$ S. C. J. Huijbregts

Leiden Institute for Brain and Cognition, and Dept. of Clinical

Child and Adolescent Studies,

Faculty of Social Sciences, Leiden University,

Wassenaarseweg 52,

2333 AK, Leiden, The Netherlands

e-mail: LdeSonneville@fsw.leidenuniv.nl

R. Licht $\cdot$ J. A. Sergeant

Dept. of Clinical Neuropsychology, VU University,

Amsterdam, The Netherlands

F. J. van Spronsen

Beatrix Children's Hospital, University Medical

Center of Groningen, University of Groningen,

Groningen, The Netherlands
Control (IDC) were associated with decreased MMN amplitudes, suggesting a reduced ability to respond to stimulus change and poorer triggering of the frontally mediated attention switch. In summary, the present study in children with PKU investigated bottom-up information processing, i.e., triggered by external events, a fundamental prerequisite for the individual's responsiveness to the outside world. Results provide evidence that quality of dietary control may affect the optimal development of these pre-attentive processes, and suggest the existence of windows of vulnerability to Phe exposure.

Keywords Phenylketonuria $\cdot$ ERP $\cdot$ VEP $\cdot$ MMN $\cdot$ dietary control $\cdot$ lifetime Phe $\cdot$ pre-attentive processing $\cdot$ attention

\section{List of abbreviations \\ AEP Auditory evoked potential \\ AOT Auditory oddball task \\ CRT Checkerboard reversal task \\ EEG Electroencephalogram \\ EOG Electro occulogram \\ ERP Event-related potential \\ IDC Index of dietary control \\ MMN Mismatch negativity \\ Phe Phenylalanine \\ PKU Phenylketonuria \\ RT Reaction time \\ Tyr Tyrosine \\ VEP Visual evoked potential}

Phenylketonuria (PKU; OMIM 261600 and 261630)) is an inherited metabolic disease that affects the metabolism of the amino acid phenylalanine (Phe). If left untreated, blood Phe levels increase and children with PKU may develop 
severe mental retardation, due to the effects of high cerebral concentrations of Phe and/or lower concentrations of other large neutral amino acids, resulting in poor myelination of neuronal connections and decreased concentration of the neurotransmitters dopamine and serotonin (Scriver et al. 1995; Van Spronsen et al. 2009). Since the discovery of effective dietary treatment (Bickel et al. 1953), prevention of mental retardation has been accomplished by early screening and the use of a low Phe diet. Although intellectual development of early-treated children with PKU is close to normal (Burgard 2000), quality of dietary treatment is critical as it has been shown that concurrent and lifetime Phe levels are negatively related to different aspects of cognitive functioning, such as executive functioning (Azadi et al. 2009; Christ et al. 2010; DeRoche and Welsh 2008; VanZutphen et al. 2007), sustained attention (Schmidt et al. 1994; Huijbregts et al. 2002a), and speed of processing (Albrecht et al. 2008).

Compromised myelination of neuronal connections, particularly in the first months of gestation, and deficient levels of dopamine and serotonin may affect the development of prefrontal cortical functions in particular (Leuzzi et al. 2000; Diamond 2001). A number of studies using magnetic resonance imaging (MRI) and/or Diffusion Tensor Imaging (DTI) indeed have shown abnormalities in the microstructural integrity of the white matter in early-treated PKU patients (Dyer 1999; Cleary et al. 1994; Thompson et al. 1993; Weglage et al. 1997), even in normal appearing white matter (Ding et al. 2008; Kono et al. 2005; Leuzzi et al. 2007; Vermathen et al. 2007; Manara et al. 2009; Scarabino et al. 2009; White et al. 2009).

Despite the evidence for (minor) structural brain deficits in PKU patients and the subtle brain dysfunctions inferred from neuropsychological assessments, more direct evidence for functional disturbances in brain areas involved in attention and information processing is scarce or inconsistent. The present study focuses on two neurophysiological markers of visual and auditory information processing: the visual evoked potential (VEP) elicited by the checkerboard reversal paradigm and the auditory mismatch negativity (MMN) elicited by an auditory oddball paradigm.

The VEP is considered to provide important information about the functional integrity of the visual system (Odom et al. 2004). The early components of the VEP to pattern reversal gratings consist of a negative peak around $75 \mathrm{~ms}$ (N75), originating from the primary visual cortex (area V1), and a positive peak around $100-110 \mathrm{~ms}$ (P100). The P100 is also generated in area $\mathrm{V} 1$, although some researchers suggest the extrastriate visual area to be a more likely candidate (Di Russo et al. 2005). Studies of pattern reversal VEPs have shown a low variability of wave form and rapid decrease in P100 latency over the first postnatal year, and VEP maturation is rapid in infants, gradual in preschool years, and persists until adulthood (Lenassi et al. 2008; Lippé et al. 2009).

Since its discovery (Näätänen et al. 1978), auditory mismatch negativity (MMN) has been linked with two important concepts of experimental psychology: memory and attention. MMN is elicited by a discriminable change in auditory stimulation (Näätänen et al. 2007). The MMN reflects an automatic process triggered by a difference between an incoming stimulus and a sensory memory trace of preceding stimuli (memory-trace hypothesis) or is thought to be generated by any irregularity that violates a pattern encoded in the auditory system (predictive models, see review by Winkler 2007). MMN is generated from auditory cortices bilaterally, possibly with an additional downstream component in the frontal cortex. The frontal MMN reflects the activation of the cortical mechanism implicated in the recruitment of attention (involuntary attention switching) (Duncan et al. 2009). To date, attempts to elucidate the role of dopaminergic and serotonergic systems on MMN have provided inconsistent results (Leung et al. 2010).

VEP latency has been shown to be longer in children with PKU than in controls (Korinthenberg et al. 1988; Ludolph et al. 1996; Leuzzi et al. 1998; Henderson et al. 2000; Koletzko et al. 2009) and was found to be correlated with diet compliance (Giovannini et al. 1988; Korinthenberg et al. 1988; Leuzzi et al. 1998), but other studies found no relationship (e.g., Pietz et al. 1993; Jones et al. 1995, in older patients). Auditory evoked potential activity has seldomly been investigated in PKU. Some studies report delayed potentials (Cardona et al. 1991; Coskun et al. 1993) while others do not (e.g. Ludolph et al. 1996).

In summary, the present study investigated bottom-up information processing, i.e., triggered by external events (vs. top-down control processes), a fundamental prerequisite for the individual's responsiveness to the outside world. Amplitude and latency of event-related potential (ERP) correlates of these automatic pre-attentive processes are thought to be affected by alterations in neuronal myelination and neurotransmitter concentrations. The goal of the present study was twofold. First, we investigated whether children with PKU differed from age-matched controls in terms of patterns of early and late ERPs elicited in response to visual and auditory stimulation. Based on evidence for both sensory and attentional deficits, it was predicted that, in comparison with healthy controls, children with PKU would show prolongation of latencies and reduction in amplitudes of ERP-waves associated with these processes. Second, we examined whether differences in long-term dietary control were related to differences in brain activity. It was predicted that indices of dietary control and concurrent Phe levels would be correlated with latencies and amplitudes of early as well as late potentials. 


\section{Methods}

The study was approved by the medical ethical committees of all participating treatment centers as well as by the Dutch National PKU Steering Committee. Written informed consent was obtained from patients' parents or caretakers.

\section{Subjects}

Sixty-seven children with PKU, sampled from the Dutch population of patients between 7 and 14 years of age $(n=85)$, participated in a national study on PKU (Huijbregts et al. 2002b). After data cleaning, the PKU group comprised 64 children (55\% female) with complete data with a mean age of $10.9(\mathrm{SD}=2.2)$ years. All diagnoses were made within the first two weeks after birth and all children with PKU were treated early $(<1$ month after birth) and continuously with dietary restrictions to limit Phe intake, and their Phe levels were monitored regularly. Blood samples were taken on the day of testing to determine concurrent Phe levels $(\mathrm{M}=$ $481 \mu \mathrm{mol} / \mathrm{L}, \mathrm{SD}=258$ ). Historical Phe levels were collected by the pediatricians with the help of TNO Prevention and Health in Leiden, The Netherlands. To study the effects of higher versus lower concurrent Phe levels, 37 children with PKU were allocated to a high Phe group (PKU-H; Phe $>360 \mu \mathrm{mol} / \mathrm{L} ; \mathrm{M}=632, \mathrm{SD}=$ 191), and 27 children were allocated to a low Phe group (PKU-L; Phe $\leq 360 \mu \mathrm{mol} / \mathrm{L} ; \mathrm{M}=226, \mathrm{SD}=101$ ). These criteria were based on treatment recommendations provided by the NIH Consensus Statement Panel (2000). Mean age of the PKU-L group was 10.98 years (SD 2.3) which did not significantly differ $(\mathrm{p}=.88)$ from the mean age of the PKU-H group of 10.90 years (SD 2.1).

The Index of Dietary Control (IDC) was computed as the mean of all half-year median blood Phe levels during the lifetime $(M=334 \mu \mathrm{mol} / \mathrm{L}, \mathrm{SD}=111)$. Mean IDC for the PKU-H group was $363 \mu \mathrm{mol} / \mathrm{L}(\mathrm{SD}=130)$, and for the PKU-L group mean IDC was $295 \mu \mathrm{mol} / \mathrm{L}(\mathrm{SD}=57)$. IDC was significantly related to concurrent Phe level $(\mathrm{r}=.53$, $\mathrm{p}<.001)$. IDC was also significantly related to all yearly Phe-means (correlations ranging from $\mathrm{r}=.48(\mathrm{p}<.001 ; 1 \mathrm{st}$ year of life) to $\mathrm{r}=.88 \quad(\mathrm{p}<.001 ; 7$ th year of life $)$. The correlations between IDC and Phe level during the first month of life (when levels are stabilized) was weaker, but still significant $(r=.34, p=.005)$. Although overall, there was a significant correlation between concurrent Phe level and IDC, concurrent Phe level was not significantly related to mean Phe level during the first month of life, or the mean Phe levels during the 1st, 4th, and 5th years of life. Similar patterns of correlations were observed for the PKU-L and the PKU-H groups, with significant associations between
IDC and Phe levels in different years (and during the first month of life), significant associations between IDC and concurrent Phe level, but no (or much weaker) relations between concurrent Phe levels and Phe levels in different years (and during the first month of life).

These descriptive statistics indicate that concurrent and lifetime Phe levels can be treated as separate (independent) predictors for the association with electrocortical activity. Further, predictions from different indices of dietary control throughout life may be theoretically informative since it is hypothesized that similar Phe levels may have a differential impact at different stages in life.

Seventy-three healthy children, aged 7-14 years, were recruited from the patients' families or peer groups or through newspaper advertisements to serve as a control group. After data cleaning, the control group comprised 69 children $(42 \%$ female) with complete data, with a mean age of $10.9(\mathrm{SD}=2.3)$ years.

\section{Tasks and stimulus material}

The checkerboard reversal task (CRT) was used to measure VEPs and consisted of the presentation of two blocks of 71 trials each. Each trial consisted of the presentation of a white and black checkerboard pattern for the duration of $400 \mathrm{~ms}$ at the center of a computer monitor. On the next trial a reversed checkerboard pattern was presented and so on. The checkerboard pattern was made up of 49 black and white checkers. Checker size was 1.65 degrees and the total display subtended 10.8 degrees. Participants were instructed to attend to the center of the computer screen during the series of trials presented and to refrain from eye blinking as much as possible.

The auditory oddball task (AOT) was used to measure auditory evoked potentials (AEP), and consisted of the presentation of series of frequent standard tones with irregular presentations of infrequent deviant tones. Nine hundred tone-trials (three blocks of 300 tone-trials each) were presented with 780 trials $(87 \%)$ consisting of standard tones $(1000 \mathrm{~Hz}), 60$ trials $(6.5 \%)$ were low deviant tones $(900 \mathrm{~Hz})$, and 60 trials $(6.5 \%)$ were high deviant tones $(1100 \mathrm{~Hz})$. The tones were presented in a pseudo-random order with at least three and maximally nine standard tones in between two deviant tones. Stimulus duration was $50 \mathrm{~ms}$ ( $5 \mathrm{~ms}$ fade-in/fade-out) and the interstimulus interval was $600 \mathrm{~ms}$, resulting into a frequency of 1.66 trials per second. Stimulus tones were presented binaurally through headphones while the participant's attention was engaged by reading a comic book. The children were instructed to pay attention only to their comic book and to discard the tones presented through the headphones. 


\section{EEG recording and procedure}

During both tasks EEG was recorded continuously from 33 AgAgCL electrodes, placed according to the 10/20 international electrode placement system (see Fig. 1), mounted in an electrode cap (ESI Electro Cap Inc). Vertical (VEOG) and horizontal (HEOG) eye movements were recorded using electrodes above and below the right eye and electrodes placed on the outer canthi of both eyes, respectively. A ground electrode was placed on the forehead and linked ear lobes were used a reference. Impedance of all electrodes was kept below $5 \mathrm{k} \Omega$.

Both EEG and EOG were pre-amplified and digitized by a portable 40 channel Twente Medical System recorder (REFA-40/DC), controlled by Poly software (Poly 4.9; Inspektor Research Systems, 1993). The time constant was set to $5 \mathrm{~s}$ and the low pass filter was set at $35 \mathrm{~Hz}$. EEG and EOG were sampled with a frequency of $200 \mathrm{~Hz}$. Stimulus presentation, timing and data logging were softwarecontrolled (ERTS 3.18, Beringer 1996).

The experiments were performed on location in the different treatment centers that participated in the study. Prior to the beginning of the EEG recordings blood samples were taken for Phe analyses. Subsequently, participants were administered a visual acuity test (Landholt ogenkaart, TNO Soesterberg, The Netherlands) and a hearing test (detection of $500 \mathrm{~Hz}, 1000 \mathrm{~Hz}$ and $2000 \mathrm{~Hz}$ probes) to detect gross visual and/or auditory deficits. The CRT was always administered first followed by the 3 blocks of the AOT that were counterbalanced across subjects.

\section{Data reduction}

The recorded continuous EEG files were analyzed using SCAN 4.2 (Neurosoft, Inc). In both tasks, EEG files were first corrected for the effects of eye-blinks using SCAN 4.2 ocular artefact correction procedures. Subsequently, the EEG files were epoched. In the checkerboard reversal task epoch length was $340 \mathrm{~ms}$ including a $50 \mathrm{~ms}$ pre-stimulus period, whereas in the auditory oddball task epoch length was $640 \mathrm{~ms}$ and included a $100 \mathrm{~ms}$ pre-stimulus period. In the next step EEG epochs were base-line corrected and trials with EEG artefacts (signals passing $\pm 100 \mu \mathrm{V}$ ) were removed. Finally, EEG and EOG signals were averaged across epochs separately for each channel and condition. In the checkerboard reversal task, average VEPs were obtained across the two blocks, whereas in the auditory oddball task average auditory evoked potentials were obtained across the three trial blocks separately for standard tones that preceded deviant tones (ST), and for the high (HD) and low deviant (LD) tones. Finally, the average pre-

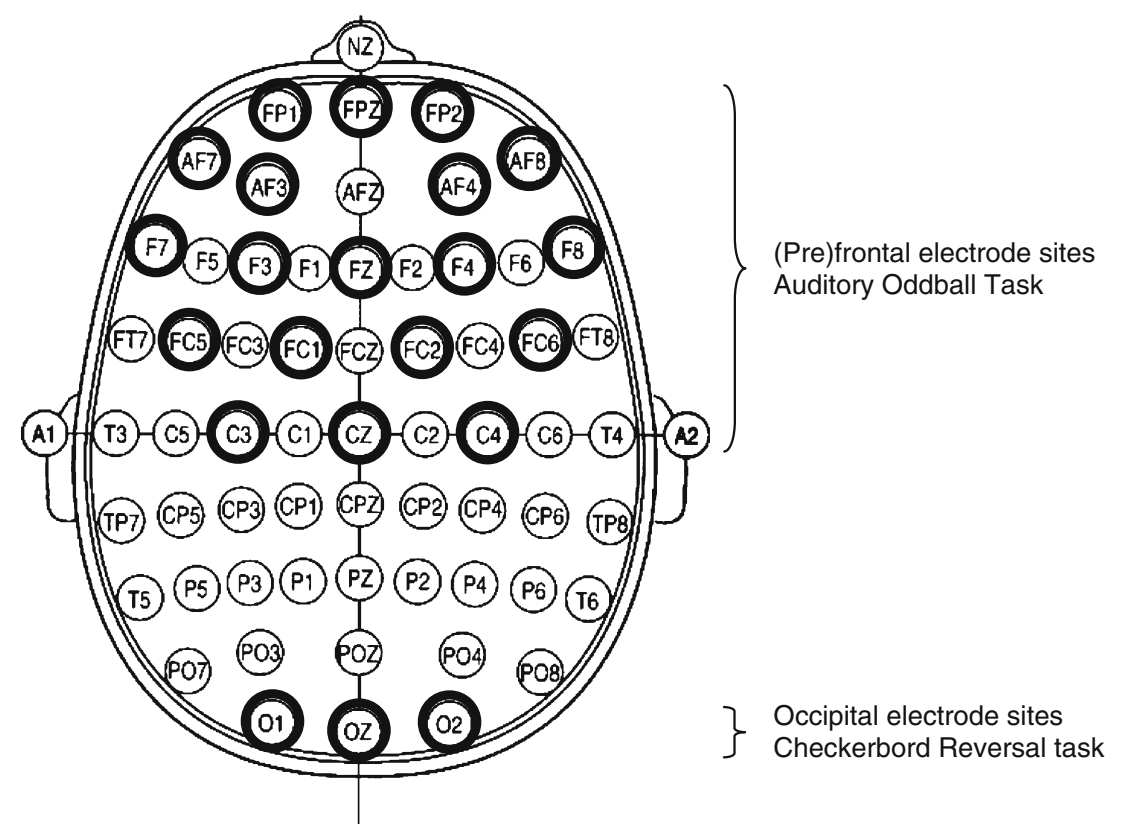

Fig. 1 The 10/20 international electrode placement system. The used electrode sites are indicated by bold circles for the Auditory oddball task (upper half) and the checker board reversal task (bottom). Each electrode position is characterized by one or more letters to identify the underlying brain area and a number or letter to identify the hemisphere (even number: right hemisphere, odd number: left hemisphere, letter $\mathrm{z}$ : midline). The letters F, C, T, P and O stand for frontal, central, temporal, parietal and occipital. The letters FP refer to a prefrontal electrode (frontopolar). Electrodes anterior to the frontal electrodes are named anterior frontal (AF). Electrodes positioned between frontal and central electrodes, central and parietal electrodes, parietal and occipital electrodes, parietal and temporal electrodes and frontal and temporal electrodes are labeled FC (frontocentral), CP (centroparietal), PO (parieto-occipital), TP (parietotemporal) and FT (frontotemporal) respectively 
stimulus baseline was subtracted from all data points in the average event related potentials (ERPs), and ERP waveforms were smoothed using a digital filter. In the auditory oddball task mismatch-related ERP waveforms (MMN) were obtained by subtracting ST ERPs from HD and LD ERPs.

To determine peak-values and latencies for both tasks, grand-average ERPs were obtained by averaging ERPs across subjects for each task separately for controls and children with PKU. Average VEPs from the CRT and average MMN waveforms in the AOT of the control sample are depicted in Figs. 2 and 3, respectively. Visual inspection of Fig. 2 shows that the VEP over occipital sites consists of an early negative peak around $75 \mathrm{~ms}$ (N75), followed by a positive peak around $110 \mathrm{~ms}(\mathrm{P} 110)$. N75 was defined as the maximum negativity at $\mathrm{O} 1, \mathrm{Oz}$ and $\mathrm{O} 2$ electrodes in the period from $50-100 \mathrm{~ms}$, whereas P110 was defined as the maximum positivity in the interval from $75-125 \mathrm{~ms}$. In addition to amplitudes, latencies of the different peaks were also determined at the occipital electrodes (see Fig. 1).

Inspection of Fig. 3 shows that the MMN in the AOT is characterized by maximal negativity at frontal sites around 190-200 ms. Due to considerable variance in MMN latencies and subsequent difficulties in peak-scoring, only MMN amplitudes were analyzed. For that purpose, average MMN amplitudes were calculated in the epoch from 150$250 \mathrm{~ms}$ at the (pre)frontal and central electrodes (see Fig. 1).

\section{Data analysis}

N75, P110 and N170 amplitudes and latencies from the CRT were analyzed using repeated measures analysis of variance (ANOVA) with Group (control, PKU-L, PKU-H) as between subjects factor and Hemisphere (O1 vs O2) as within subjects factor. Amplitudes and latencies of the same

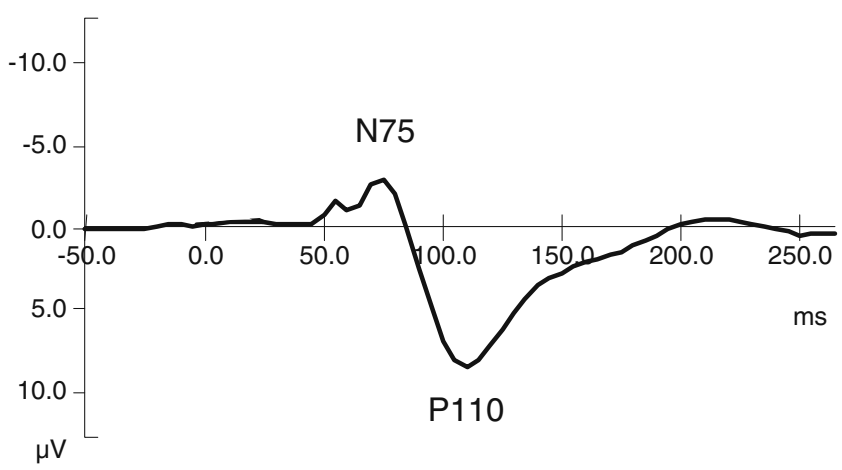

Fig. 2 Averaged visual evoked potential of control sample at the midline occipital electrode site $(\mathrm{Oz})$. N75=maximum negative peak at $75 \mathrm{~ms}$ post stimulus onset. $\mathrm{P} 110=$ maximum positive peak at $110 \mathrm{~ms}$ post stimulus onset

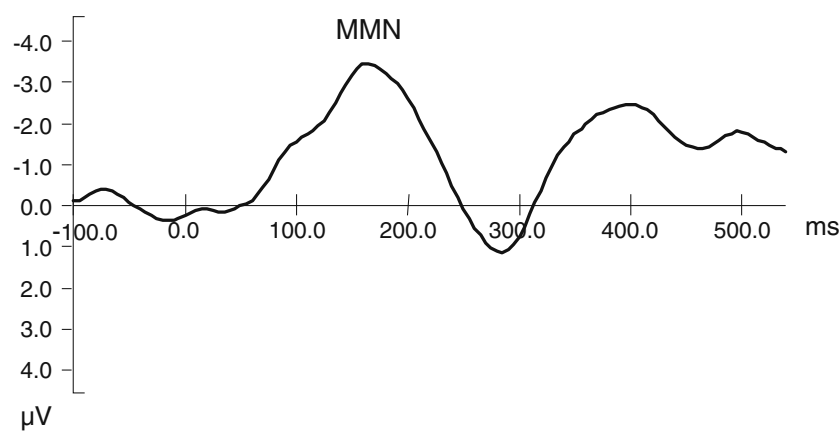

Fig. 3 Averaged mismatch negativity (MMN) of control sample at the midline frontal electrode site $(\mathrm{Fz})$

peaks at $\mathrm{Oz}$ were analyzed using a One Way ANOVA with Group as between subjects factor. MMN amplitudes from the AOT were analyzed employing repeated measures ANOVA with Group as between subject factor and Topography (outer lateral vs inner lateral sites) and Hemisphere (left vs. right) as within subjects factors. For all analyses involving factors with more than two levels Greenhouse-Geisser corrected p-values will be reported to prevent inflation of statistical significance.

Pearson product-moment correlations were computed and regression analyses were performed to assess the relationship between variance found in the total sample in Phe levels (concurrent, lifetime and IDC) and the variances in indices of brain activity. Life time Phe levels were the median of Phe recordings for each year from 0 to 10 years of age. The IDC was represented by the average median Phe level across the first 10 years (or less if children were younger). Age was always entered as the first variable in these analyses. Regression results will be reported only if standardized residuals were below three and Cook's distances were below one (Stevens 1996). Effect sizes were estimated using partial eta squared $\left(\eta_{\mathrm{p}}{ }^{2}\right)$, and interpreted as small $\left(\eta_{\mathrm{p}}{ }^{2} \approx 0.01\right)$, medium $\left(\eta_{\mathrm{p}}{ }^{2} \approx \mathrm{d} 0.06\right)$, and large $\left(\eta_{\mathrm{p}}{ }^{2}>\right.$ 0.13) (Cohen 1988).

\section{Results}

Effects of changes in visual stimulation on VEP

Analyses of N75 and P110 amplitudes and latencies at O1 and $\mathrm{O} 2$, and at $\mathrm{Oz}$ respectively, did not reveal any significant main effect or interaction, indicating that controls and children with PKU, and children with PKU-L and PKU-H, respectively, did not differ in occipital amplitudes and latencies.

Significant correlations, however, were found between concurrent Phe levels and P110 latencies at $\mathrm{O} 2$ $(\mathrm{r}=.25, p=.023)$ and $\mathrm{N} 170$ latencies at $\mathrm{Oz}(\mathrm{r}=.21$, 
$p=.048)$, and between IDC scores and $\mathrm{N} 170$ at $\mathrm{Oz}(\mathrm{r}=.25$, $p=.038)$, indicating that latencies increase with increasing Phe or IDC levels. The analyses of historical levels revealed that P110 amplitude at $\mathrm{O} 1$ was associated with median Phe levels at ages 2 and 4-7 years ( $\mathrm{r}$ varies from .26 to .28, $p$-values vary from .025 to $.018,1$-tailed). At age $9, \mathrm{~N} 75$ amplitudes at $\mathrm{O} 1, \mathrm{O} 2$ and $\mathrm{Oz}$ correlated significantly with Phe levels ( $\mathrm{r}$ varies from -.33 to -.37 , $\mathrm{p}$-values vary from .014 to .006, 1-tailed). N75 latencies at $\mathrm{O} 1, \mathrm{O} 2$, and $\mathrm{Oz}$ correlated with Phe levels at age 4-7 years ( $\mathrm{r}$ varies from .225 to $-.33, p$-values vary from .042 to $.007,1$-tailed). In sum, these correlations indicate that higher life time Phe levels at younger ages were associated with larger P110 latencies and amplitudes, larger N75 amplitudes and shorter N75 latencies.

Regression analyses with Phe levels as independent variables and VEP latencies and amplitudes as dependent variables (see Table 1) confirmed these findings. Variance in concurrent Phe levels explained $4.8 \%$ of variance in $\mathrm{P} 110$ latencies at $\mathrm{O} 2$. The analyses on lifetime data showed that Phe levels at age 4, 5, and 6 years accounted for $11.9 \%$, $25 \%$, and $14.5 \%$ of variance, respectively, in P110 amplitudes at $\mathrm{O} 1, \mathrm{O} 2$, and $\mathrm{Oz}$. In addition, Phe levels at age 4 and 5 years accounted for $5.6 \%$ and $15.1 \%$ of variance in N75 latencies respectively, whereas Phe levels at age 9 years explained $11.7 \%$ of the variance in N75 amplitudes at $\mathrm{O} 2$.

\section{Effects of changes in auditory stimulation (MMN)}

Analyses of the MMN amplitudes at the selected lateral frontal sites showed that MMN was larger at F3/F4 than at $\mathrm{F} 7 / \mathrm{F} 8\left(\mathrm{~F}(1,104)=9.488, p=.003, \eta^{2}=.084\right)$, and was larger over the right than over the left hemisphere at frontal $(\mathrm{F})$ and fronto-central $(\mathrm{FC})$ sites $(\mathrm{F}(1,104)=7.229, p=.008$, $\left.\eta^{2}=.065 ; \mathrm{F}(1,105)=6.585, p=.012, \eta^{2}=.059\right)$. No significant Group effects or interactions involving Group were found, indicating that controls and children with PKU, and children with PKU-L and PKU-H, respectively, did not differ in MMN latency and amplitude.

Multiple regression analyses on MMN amplitudes and concurrent Phe levels and IDCs (see Table 2) revealed that variance in IDC scores accounted for $8.1 \%$ of the variance in MMN amplitudes elicited by low tone deviants, and for $52.2 \%$ of the variance in average MMN amplitudes (mean of low deviant tone MMN and high deviant tone MMN) at frontal sites. Variance in concurrent Phe levels explained $14.8 \%$ of variance in average MMN amplitudes at frontal sites.

Correlations between Phe and IDC and MMN amplitudes for low tone deviants and average MMN amplitudes at AF8 and F4 ranged from .29 to .40 ( $p$ ranges from .023 to .004 , 1-tailed), indicating that increased Phe or IDC scores were associated with reduced MMN amplitudes.

More detailed stepwise regression analyses on lifetime Phe levels showed that individual differences in Phe levels in the first year of life accounted for $11.6 \%$ of the variance in average MMN amplitudes, and for $33.3 \%$ of variance in high tone MMN amplitudes at frontal and anterior-frontal sites. MMN amplitudes elicited by low tone deviants accounted for $12.1 \%$ of the variance in Phe levels at age 2 years. Further significant relationships were found between Phe levels at age 8 years and high tone MMN amplitudes at frontal sites (18.2\% of variance explained) and between Phe levels at age 9 years and high tone MMN amplitudes at right frontal sites (39.1\%), and low tone MMN amplitudes at fronto-central midline sites (35.4\%). Zero-order correlations between lifetime Phe levels and MMN amplitudes for significant regression effects ranged from .30 to .52 ( $p$-values ranged from .024 to $.0001,1$ tailed). All correlations indicate that increased Phe levels at younger and older ages are associated with reduced MMN amplitudes.
Table 1 Results of regression analyses on concurrent and lifetime Phe measures and VEP amplitudes (amp) and latencies (lat). Regression results are reported only if standardized residuals were below three and Cook's distances were below one and the regression model was significant. $\mathrm{R}^{2}=$ portion of explained variance in the VEP components. For example: Phe levels at the age of 5 accounted for $25 \%$ (.0252) of the variance in P110 amplitude at the occipital sites

\begin{tabular}{|c|c|c|c|c|c|c|}
\hline Phe measure & VEP component & Sites & $\mathrm{R}^{2}$ & $\mathrm{df}$ & $\mathrm{F}$ & $\mathrm{P}$ \\
\hline Conc. Phe & P110 lat & $\mathrm{O} 2$ & .048 & $1 / 61$ & 4.05 & .049 \\
\hline \multirow[t]{2}{*}{ Phe age 4} & P110 amp & $\mathrm{O} 1, \mathrm{O} 2, \mathrm{Oz}$ & .119 & $2 / 51$ & 4.44 & .017 \\
\hline & N75 lat & $\mathrm{O} 2$ & .056 & $1 / 51$ & 4.04 & .050 \\
\hline \multirow[t]{2}{*}{ Phe age 5} & P110 amp & $\mathrm{O} 1, \mathrm{O} 2, \mathrm{Oz}$ & .252 & $2 / 52$ & 9.68 & .001 \\
\hline & N75 lat & $\mathrm{O} 1, \mathrm{O} 2, \mathrm{Oz}$ & .151 & $2 / 53$ & 5.64 & .006 \\
\hline Phe age 6 & P110 amp & $\mathrm{O} 1, \mathrm{O} 2, \mathrm{Oz}$ & .145 & $2 / 53$ & 5.51 & .007 \\
\hline Phe age 9 & N75 amp & $\mathrm{O} 2$ & .117 & $1 / 41$ & 6.55 & .014 \\
\hline
\end{tabular}


Table 2 Results of regression analyses on concurrent and lifetime Phe measures, IDC and MMN amplitudes. $1 \mathrm{t}=$ low tone deviants, ht=high tone deviants, $\mathrm{av}=$ average $\mathrm{MMN}$ amplitudes.

Regression results are reported only if standardized residuals were below 3 and Cook's distances were below 1 and the regression model was significant. $\mathrm{R}^{2}=$ portion of explained variance in the MMN components

\begin{tabular}{lllllll}
\hline Phe measure & MMN component & Sites & $\mathrm{R}^{2}$ & $\mathrm{df}$ & $\mathrm{F}$ & $\mathrm{P}$ \\
\hline Conc.Phe & MMN-av & AF8, F8 & .148 & $2 / 45$ & 5.07 & .010 \\
IDC & MMN-lt & F & .081 & $1 / 41$ & 4.73 & .036 \\
ln(IDC) & MMN-av & AF3, AF4, Fpz, Fp2 & .522 & $4 / 39$ & 12.74 & .0001 \\
Phe age 1 & MMN-av & F, AF & .116 & $1 / 41$ & 6.53 & .014 \\
& MMN-ht & F, AF & .333 & $2 / 40$ & 11.50 & .0001 \\
Phe age 2 & MMN-lt & F, AF & .121 & $1 / 40$ & 6.67 & .014 \\
Phe age 8 & MMN-ht & FC6, FPz & .182 & $2 / 38$ & 5.47 & .008 \\
Phe age 9 & MMN-ht & F8, Fp2 & .391 & $2 / 33$ & 12.21 & .0001 \\
& MMN-lt & Cz, Fz, Fpz & .354 & $3 / 32$ & 7.39 & .001 \\
\hline
\end{tabular}

life than for concurrent Phe levels. Long lasting effects of first month Phe levels, ranging from $1050-1470 \mu \mathrm{mol} / \mathrm{L}$, on visual contrast sensitivity in children with PKU, with a mean age 7.1 years $(\mathrm{SD}=1.1)$, have been reported by Diamond and Herzberg (1996), suggesting some early structural damage to the visual system, possibly due to elevated Phe levels in the first post-natal weeks. In the present study, post-natal Phe levels, measured within 2 weeks after birth were also highly elevated (about $1350 \mu \mathrm{mol} / \mathrm{L}$ ) which might have interfered with a normal development of the dopamine-sensitive visual system. We did however not find significant predictions of VEPcomponents from these early Phe levels. There was a significant prediction from concurrent Phe, suggesting a role for dopamine, but predictions from several lifetime measures were generally stronger, which might suggest 1) a weaker-than-expected role in this task for dopaminedependent visual information processing, and 2) a largerthan-expected role for cortical structures (i.e., the occipital lobe) in the type of visual bottom-up processing associated with this task. Although neural proliferation ends at birth, apoptosis continues well into the first years of life, and synaptogenesis and myelination continue into adolescence and adulthood, respectively (Tau and Peterson 2010). Myelination and synaptic remodeling are particularly active between the ages of 2 and 5, and cortical gray matter continues to increase in volume through the ages of 4 or 5 years (Levitt 2003). Thus, it may be the case that structural damage to cortical structures following prolonged periods of elevated Phe may also affect pre-attentive visual processing. Our data also appear to indicate that Phe levels representative of dietary control throughout life (considering the high correlations between different indices) are more harmful during specific critical periods in brain development.

Pre-attentive auditory processing

The AOT showed the expected maximal distribution of MMN over frontal sites with larger amplitudes over the right than over the left hemisphere (Giard et al. 1990). explanatory power for Phe levels during specific periods of 
Although we predicted smaller MMN amplitudes for children with PKU compared with normal controls, in particular for children with PKU with high Phe levels, no direct support for this hypothesis was found from the analyses of variance. However, positive correlations between MMN amplitudes and IDC and Phe levels revealed that higher IDC scores and Phe levels were associated with reduced MMN amplitudes, indirectly confirming our hypothesis that elevated Phe levels would result in smaller MMN amplitudes. Smaller MMN amplitudes have been reported to indicate reduced ability to detect stimulus change and poorer triggering of the frontally mediated attention switch (Näätänen 1990). Multiple regression analyses showed that variance in IDC scores accounted for $52 \%$ of the variance in average MMN amplitudes at frontal sites. Moreover, detailed stepwise regression analyses on lifetime Phe levels showed that individual differences in Phe level in the first two years of life accounted for 11.6 of the variance in average MMN amplitudes and for $33.3 \%$ in high tone MMN amplitudes. These findings are intriguing and suggest that individual differences in Phe levels in the first two years of life exert their influence on frontal brain activity (in this case MMN responses) over a period of at least 6-12 years. Similar long-lasting relationships between lifetime Phe levels and VEP amplitudes and latencies were found, but at a later period from 4-6 years. In the MMN-task, however, there was also a significant prediction of high tone MMN amplitudes at frontal and anterior-frontal sites from Phe level at age 8-9 years. Diffusion tensor imaging (DTI) studies show that white matter organization continues to develop into adolescence, although its rate of increase is highest before the age 10 (Ashtari et al. 2007; Giorgio et al. 2008). The prediction of MMN amplitudes from Phe levels at age 8-9 years might be indicative of Phe-related infringements of this process of white matter organization.

In summary, the present study investigated bottom-up information processing, i.e., triggered by external events, a fundamental prerequisite for the individual's responsiveness to the outside world. Overall, the results confirm the notion that the quality of dietary control may affect the development and maturation of neural circuits, in particular when passing through critical periods of vulnerability and opportunity.

The fact that these effects exist over such a long-time period could indicate that the assumed structural damage due to early highly elevated Phe levels (Diamond and Herzberg 1996) may have affected the normal development of frontal brain functioning in particular. Anderson et al. (2004) found lifetime Phe levels to be correlated with severity of white matter abnormalities in children with PKU, explaining its stronger association with cognitive performance than concurrent Phe levels. In addition, it has been suggested that even small reductions in tyrosine, a precursor of dopamine, has detrimental effects especially on the dopamine system in pre-frontal brain areas (e.g., dorsolateral prefrontal cortex) that may lead to considerable cognitive deficits (Diamond 2001). Interestingly, there is a growing body of evidence that children with PKU indeed have difficulties performing executive function tasks that are primarily mediated by (pre)frontal brain areas and that involve, e.g., attentional control, response switching, and monitoring (Diamond et al. 1997; Huijbregts et al. 2002a; Leuzzi et al. 2004; Stemerdink et al. 1999; Weglage et al. 1996; Welsh et al. 1990), although it must be noted that evidence has been presented lately, that does not support the executive deficit hypothesis (Channon et al. 2004, 2005). The smaller MMN amplitudes found for children with PKU with higher concurrent and elevated early lifetime Phe levels could be an indication of frontal dysfunctions that may underlie executive deficits later in childhood/adolescence when processing skills call more strongly upon intact frontal brain areas (Kolb and Fantie 1997; Diamond 2001).

There are limitations to our study. This study is inevitably about many electrode sites and historical Phelevels. Therefore, it was tried to capture possible relationships between Phe-levels and electrocortical indices with multiple regression analysis, combining sites. Many strong multiple correlations were found, the majority reaching Pvalues $<.01$. Additionally, one could opt to apply a Bonferroni correction to adjust for multiple testing, but, in particular because values on neighboring electrode sites are known to be correlated, we agree with Perneger (1998) to not going this way, but simply describe what we see.

Although our group of patients with PKU was relatively large, statistical analyses of the many conditions of the task, as well as partitioning into high Phe $(>360 \mu \mathrm{mol} / \mathrm{L})$ and low Phe $(\leq 360 \mu \mathrm{mol} / \mathrm{L})$ subgroups, and the substantial age range, would have benefited from a larger sample. Also, it would have been beneficial to a theoretical account of our results if we had included ERP-measurements during topdown information processing. Requirement of cognitive control could have revealed group differences in electrocortical measures equivalent to group differences observed in neuropsychological testing of cognitive control. Finally, future studies could consider the use of more refined measures of dietary control when predicting electrocortical (or neuropsychological) outcomes, such as phe:tyr ratio and measures of individual phe-level variations across time.

Details of funding This work was supported by ZorgOnderzoek Nederland ( $\mathrm{nr} 28-2719)$. The authors confirm independence from the above sponsor; the content of the article has not been influenced by the sponsor. 
Open Access This article is distributed under the terms of the Creative Commons Attribution Noncommercial License which permits any noncommercial use, distribution, and reproduction in any medium, provided the original author(s) and source are credited.

\section{References}

Agostoni C, Harvie A, McCulloch DL, Demellweek C, Cockburn F, Giovannini M, Murray G, Harkness RA (2006) A randomized trial of long-chain polyunsaturated fatty acid supplementation in infants with phenylketonuria. Dev Med Child Neurol 48:207212

Albrecht J, Garbade SF, Burgard P (2008) Neuropsychological speed tests and blood phenylalanine levels in patients with phenylketonuria: a meta-analysis. Neurosci Biobehav Rev 33:414-421

Anderson PJ, Wood SJ, Francis DE, Coleman L, Warwick L, Casanelia S, Anderson VA, Boneh A (2004) Neuropsychological functioning in children with early-treated phenylketonuria: Impact of white matter abnormalities. Dev Med Child Neurol 46:230-238

Ashtari M, Cervellione KL, Hasan KM, Wu J, McIlree C, Kester H et al. (2007) White matter development during late adolescence in healthy males: a cross-sectional diffusion tensor imaging study. Neuroimage 35:501-510

Azadi B, Seddigh A, Tehrani-Doost M, Alaghband-Rad J, Ashrafi MR (2009) Executive dysfunction in treated phenylketonuric patients. Eur Child Adolesc Psychiatry 18:360-368

Beblo S, Reinhardt A, Muntau AC, Mueller-Felber W, Roscher A, Koletzko B (2001) Fish oil supplementation improves visual evoked potentials in children with phenylketonuria. Neurology 57:1488-1491

Beringer J (1996) Experimental Run Time Systems. BeriSoft Cooperation, Frankfurt an Main, Germany

Bickel H, Gerrard J, Hickmans EM (1953) Influence of phenylalanine intake on phenylketonuria. Lancet 265:812-813

Burgard P (2000) Development of intelligence in early treated phenylketonuria. Eur J Pediatr 159(Suppl 2):S74-S79

Cardona F, Leuzzi V, Antonozi I, Benedetti P, Loizzo A (1991) The development of auditory and visual evoked potentials in early treated phenylketonuric children. EEG Clin Neurophysiol 80:815

Channon S, German E, Cassina C, Lee P (2004) Executive functioning, memory and learning in phenylketonuria. Neuropsychology 18:613-620

Channon S, Mockler C, Lee P (2005) Executive functioning and speed of processing in phenylketonuria. Neuropsychology 18:679-686

Christ SE, Huijbregts SCJ, De Sonneville LMJ, White DA (2010) Executive Function in Early-Treated Phenylketonuria: Profile and Underlying Mechanisms. Mol Genet Metab 99:S22-S32

Cleary MA, Walter JH, Jenkins JP, Alani SM, Tyler K, Whittle D (1994) Magnetic resonance imaging of the brain in phenylketonuria. Lancet 344:87-90

Cohen J (1988) Statistical power analysis for the behavioral sciences. Lawrence Erlbaum Associates, Hillsdale, NJ

Coskun T, Topcu M, Ustundag I, Ozlap I, Renda Y, Cyger A, Nurlu G (1993) Neurophysiological studies of patients with classical phenylketonuria: Evaluation of results of IQ scores, EEG and evoked potentials. Turk J Pediatr 35:1-10

Creel D, Buehler BA (1982) Pattern evoked potentials in phenylketonuria. EEG Clin Neurophysiol 53:220-223

DeRoche K, Welsh M (2008) Twenty-five years of research on neurocognitive outcomes in early-treated phenylketonuria: intelligence and executive function. Dev Neuropsychol 33:474-504
Di Russo F, Pitzalis S, Spitoni G, Aprile T, Patria F, Spinelli D, Hillyard SA (2005) Identification of the neural sources of the pattern-reversal VEP. Neuroimage 24:874-886

Diamond A (2001) A model system for studying the role of dopamine in the prefrontal cortex during esrly development in humans: Early and continuously treated phenylketonuria. In: Nelson CA, Luciana M (eds) Handbook of developmental cognitive neuroscience. Massachusetts, MIT Press, Cambridge

Diamond A, Herzberg C (1996) Impaired sensitivity to visual contrast in children treated early and continuously for phenylketonuria. Brain 119:523-538

Diamond A, Prevor MB, Callender G, Druin DP (1997) Prefrontal cortex cognitive deficits in children treated early and continuously for PKU. Monographs Soc Res Child Develop 62 (4, Serial No. 252)

Ding XQ, Fiehler J, Kohlschutter B, Wittkugel O, Grzyska U, Zeumer $\mathrm{H}$, Ullrich K (2008) MRI abnormalities in normal-appearing brain tissue of treated adult PKU patients. J Magn Reson Imaging 27:998-1004

Duncan CC, Barry RJ, Conolly JF, Fischer C, Michie PT, Näätänen R, Polich J, Reinvang I, Van Petten C (2009) Event-related potentials in clinical research: guidelines for eliciting, recording, and quantifying mismatch negativity, P300, and N400. Clin Neurophysiol 120:1883-1908

Dyer C (1999) Pathophysiology of phenylketonuria. Ment Retard Dev Disabil Res Rev 5:104-112

Giard MH, Perrin F, Pernier J, Bouchet P (1990) Brain generators implicated in processing of auditory stimulus deviance: a topographic event-related potential study. Psychophysiology 27:627-740

Giorgio A, Watkins KE, Douaud G, James AC, James S, De Stefano $\mathrm{N}$ et al. (2008) Changes in white matter microstructure during adolescence. Neuroimage 39:52-61

Giovannini M, Valsasina R, Villani R, Ducati A, Riva E, Landi A, Longhi R (1988) Pattern reversal visual evoked potentials in phenylketonuria. J Inherit Metab Dis 11:416-421

Henderson RM, McCulloch DL, Herbert AM, Robinson PH, Taylor MJ (2000) Visual event-related potentials in children with phenylketonuria. Acta Paediatr 89:52-57

Hommes FA, Eller AG, Taylor EH (1982) Turnover of the fast components of myelin and myelin proteins in experimental hyperphenylalanaemia. Relevance to the termination of dietary treatment in human phenylketonuria. J Inherit Metab Dis 5:21-27

Huijbregts SCJ, De Sonneville LMJ, Licht R, Van Spronsen FJ, Verkerk PH, Sergeant JA (2002a) Sustained attention and inhibition of cognitive interference in treated phenylketonuria: Associations with concurrent and lifetime phenylalanine concentrations. Neuropsychologia 40:7-15

Huijbregts SCJ, De Sonneville LMJ, Van Spronsen FJ, Licht R, Sergeant JA (2002b) The neuropsychological profile of early and continuously treated phenylketonuria: Orienting, vigilance, and maintenance versus manipulation-functions of working memory. Neurosci Biobehav Rev 26:697-712

Jones SJ, Turano G, Kriss A, Shawkat F, Kendall B, Thompson AJ (1995) Visual evoked potentials in phenylketonuria: association with brain MRI, dietary state, and IQ. J Neurol Neurosurg Psychiatry 59:260-265

Kolb B, Fantie B (1997) Development of the child's brain and behavior. In: Reynolds CR, Fletcher-Janzen E (eds) Handbook of clinical child neuropsychology. Plenum, New York, pp 1741

Koletzko B, Beblo S, Demmelmair H, Müller-Felber W, Hanebutt FL (2009) Does dietary DHA improve neural function in children? Observations in phenylketonuria. Prostaglandins Leukot Essent Fatty Acids 81:159-164 
Kono K, Okano Y, Nakayama K, Hase Y, Minamikawa S, Ozawa N, Yokote N, Inoue Y (2005) Diffusion-weighted MR imaging in patients with phenylketonuria: relationship between serum phenylalanine levels and ADC values in cerebral white matter. Radiology 236:630-636

Korinthenberg R, Ullrich K, Füllenkemper F (1988) Evoked potentials and electroencephalography in adolescents with phenylketonuria. Neuropediatrics 19:175-178

Lenassi E, Likar K, Stirn-Kranjc B, Brecelj J (2008) VEP maturation and visual acuity in infants and preschool childern. Doc Ophthalmol 117:111-120

Leung S, Croft RJ, Guille V, Scholes K, O’Neill BV, Phan KL, Nathan PJ (2010) Acute dopamine and/or serotonin depletion does not modulate mismatch negativity (MMN) in healthy human participants. Psychopharmacology 208:233-244

Leuzzi V, Rinalduzzi S, Chiarotti F, Garzia P, Trasimeni G, Accornero N (1998) Subclinical visual impairment in phenylketonuria. A neurophysiological study (VEP-P) with clinical, biochemical, and neuroradiological (MRI) correlations. J Inherit Metab Dis 21:351-364

Leuzzi V, Seri S, Cerquiglini A, Cl C, Ca C, Antonozzi I (2000) Derangement of the dopaminergic system in phenylketonuria: Study of the event-related potential (P300). J Inherit Metab Dis 23:317-320

Leuzzi V, Pansini M, Sechi E, Chiarotti F, Carducci C, Levi G, Antonozzi I (2004) Executive function impairment in earlytreated PKU subjects with normal mental development. J Inherit Metab Dis 27:115-125

Leuzzi V, Tosetti M, Montanaro D, Carducci C, Artiola C, Carducci C, Antonozzi I, Burroni M, Carnevale F, Chiarotti F, Popolizio P, Giannatempo GM, D'Alesio V, Scarabino T (2007) The pathogenesis of the white matter abnormalities in phenylketonuria. A multimodal 3.0 tesla MRI and magnetic resonance spectroscopy (1 H MRS) study. J Inherit Metab Dis 30:209-216

Levitt P (2003) Structural and functional maturation of the developing primate brain. J Pediatr 143:S35-S45

Lippé S, Kovacevic N, McIntosh AR (2009) Differential maturation of brain signal complexity in the human auditory and visual system. Front Hum Neurosci 3(48):1-9. doi:10.3389/neuro.09.048.2009

Ludolph AC, Vetter U, Ullrich K (1996) Studies of multimodal evoked potentials in treated phenylketonuria: the pattern of vulnerability. Eur J Pediatr 15(suppl 1):S64-S68

Manara R, Burlina AP, Citton V, Ermani M, Vespignani F, Carollo C, Burlina AB (2009) Brain MRI diffusion-weighted imaging in patients with classical phenylketonuria. Neuroradiology. doi:10.1007/s00234-009-0574-z

Näätänen R (1990) The role of attention in auditory information processing as revealed by event-related potentials and other brain measures of cognitive function. Behav Brain Sci 13:201-288

Näätänen R, Gaillard AWK, Mäntysalo S (1978) Early selective attention effect on evoked potential reinterpreted. Acta Psychol 42:313-329

Näätänen R, Paavilainen P, Rinne T, Alho K (2007) The mismatch negativity (MMN) in basic research of central auditory processing: a review. Clin Neurophysiol 118:2544-2590

Odom JV, Bach M, Barber C, Brigell M, Marmor MF, Tormene AP, Holder GE (2004) Visual evoked potentials standard. Doc Ophthalmol 108:115-123

Perneger TV (1998) What's wrong with Bonferroni adjustments. BMJ 316:1236-1238

Pietz J, Schmidt E, Matthis P, Kobialka B, Kutscha A, De Sonneville LMJ (1993) EEGs in phenylketonuria. I: Follow-up to adulthood;
II: Short-term diet-related changes in EEGs aand cognitive function. Dev Med Child Neurol 35:54-64

Scarabino T, Popolizio T, Tosetti M, Montanaro D, Giannatempo GM, Terlizzi R, Pollice S, Maiorana A, Maggialetti N, Carriero A, Leuzzi V, Salvolini U (2009) Phenylketonuria: white-matter changes assessed by 3.0-T magnetic resonance (MR) imaging, MR spectroscopy and MR diffusion. Radiol Med 114:461-474

Schmidt E, Rupp A, Burgard P, Pietz J, Weglage J, De Sonneville LMJ (1994) Sustained attention in adult phenylketonuria: The influence of the concurrent phenylalanine-blood-level. J Clin Exp Neuropsychol 16:681-688

Scriver CR, Eisensmith RC, Woo SLC (1995) The hyperphenylalaninemias, In: The Metabolic and Molecular Bases of Inherited Disease, 7th edn. McGraw-Hill, New York, pp 1015-1075

Stemerdink NBA, Van der Molen MW, Kalverboer AF, Van der Meere JJ, Huisman J, De Jong LWA, Slijper FME, Verkerk PH, Van Spronsen FJ (1999) Prefronal dysfunction in early and continuously treated phenylketonuria. Develop Neuropsychol 16:29-57

Stevens J (1996) Applied multivariate statistics for the social sciences. Lawrence Erlbaum, Mahwah, NJ

Tau GZ, Peterson BS (2010) Normal development of brain circuits. Neuropsychopharmacol Rev 35:147-168

Thompson AJ, Smith I, Brenton D, Youl BD, Rylance G, Davidson DC, Kendall B, Lees AJ (1990) Neurological deterioration in young adults with phenylketonuria. Lancet 336:602-605

Thompson AJ, Tillotson S, Smith I, Kendall B, Moore SG, Brenton DP (1993) Brain MRI changes in phenylketonuria. Associations with dietary status. Brain 116:811-821

Ullrich K, Weglage J, Oberwittler C, Pietsch M, Funders B, Eckhardstein HV (1996) Effect of L-Dopa on visual evoked potentials and neuropsychological tests in adult phenylketonuria patients. Eur J Pediatr 155(Suppl 1):S74-S77

Van Spronsen FJ, Hoeksma M, Reijngoud DJ (2009) Brain dysfunction in phenylketonuria: is phenylalanine toxicity the only possible cause? J Inherit Metab Dis 32:46-51

VanZutphen KH, Packman W, Sporri L, Needham MC, Morgan C, Weisiger K, Packman S (2007) Executive functioning in children and adolescents with phenylketonuria. Clin Genet 72:13-18

Vermathen P, Robert-Tissot L, Pietz J, Lutz T, Boesch C, Kreis R (2007) Characterization of white matter alterations in phenylketonuria by magnetic resonance relaxometry and diffusion tensor imaging. Magn Reson Med 58:1145-1156

Weglage J, Pietsch M, Funders B, Koch HG, Ullrich K (1996) Deficits in selective and sustained attention processes in early treated children with phenylketonuria: Result of impaired frontal lobe functions? Eur J Pediatr 155:200-2004

Weglage J, Bick U, Schuierer G, Pietsch M, Sprinz A, Zab R, Ullrich K (1997) Progression of cerebral white matter abnormalities in early-treated patients with phenylketonuria during adolescence. Neuropediatrics 28:239-240

Welsh MC, Pennington BF, Ozonoff S, Rouse B, McCabe ERB (1990) Neuropsychology of early-treated phenylketonuria: specific executive function deficits. Child Dev 61:1697-1713

White D, Connor L, Nardos B, Shimony J, Archer R, Synder A, Moinuddin A, Grange D, Steiner R, McKinstry R (2009) Agerelated decline in the microstructural integrity of white matter in children with early- and continuously-treated phenylketonuria: a diffusion tensor imaging study of the corpus callosum. Mol Genet Metab 99:S41-S46

Winkler I (2007) Interpreting the mismatch negativity. J Psychophysiol 21:147-163 